\title{
Empirical Distributions of Laplacian Matrices of Large Dilute Random Graphs
}

\author{
TIEFENG JiAnG ${ }^{1}$
}

\begin{abstract}
We study the spectral properties of the Laplacian matrices and the normalized Laplacian matrices of the Erdös-Rényi random graph $G\left(n, p_{n}\right)$ for large $n$. Although the graph is simple, we discover some interesting behaviors of the two Laplacian matrices. In fact, under the dilute case, that is, $p_{n} \in(0,1)$ and $n p_{n} \rightarrow \infty$, we prove that the empirical distribution of the eigenvalues of the Laplacian matrix converges to a deterministic distribution, which is the free convolution of the semicircle law and $N(0,1)$. However, for its normalized version, we prove that the empirical distribution converges to the semi-circle law.
\end{abstract}

\section{Introduction}

In graph theory, the Erdös-Rényi model $G(n, p)$, named for Erdös-Rényi [17, 18, 19, 20], is a graph with $n$ vertices; a potential edge between each pair of vertices has probability $p$, independently of the other edges. Some properties of $G(n, p)$ such as the diameters, the sizes and the giant components are known, see, e.g., $[5,9,17,18,19,20]$ for details. For the spectral properties of the graphs, one can see, e.g., $[9,10,11,15,27,31]$.

The graph $G\left(n, p_{n}\right)$ corresponds to Bernoulli random variables $\left\{\xi_{i j}^{(n)} ; 1 \leq i<j \leq n\right\}$, which are independent random variables with $\xi_{i j}^{(n)}=\xi_{j i}^{(n)}$ and $P\left(\xi_{i j}^{(n)}=1\right)=1-P\left(\xi_{i j}^{(n)}=0\right)=p_{n}$ for all $1 \leq i<j \leq n$. The adjacency matrix $\mathbf{A}_{n}$ and Laplacian matrix $\boldsymbol{\Delta}_{n}$ are defined as follows:

$$
\mathbf{A}_{n}=\left(\begin{array}{cccc}
0 & \xi_{12}^{(n)} & \cdots & \xi_{1 n}^{(n)} \\
\xi_{21}^{(n)} & 0 & \cdots & \xi_{2 n}^{(n)} \\
\vdots & \vdots & \vdots & \vdots \\
\xi_{n 1}^{(n)} & \xi_{n 2}^{(n)} & \cdots & 0
\end{array}\right)
$$

and

$$
\boldsymbol{\Delta}_{n}=\left(\begin{array}{cccc}
\sum_{j \neq 1} \xi_{1 j}^{(n)} & -\xi_{12}^{(n)} & \cdots & -\xi_{1 n}^{(n)} \\
-\xi_{21}^{(n)} & \sum_{j \neq 2} \xi_{2 j}^{(n)} & \ldots & -\xi_{2 n}^{(n)} \\
\vdots & \vdots & \vdots & \vdots \\
-\xi_{n 1}^{(n)} & -\xi_{n 2}^{(n)} & \cdots & \sum_{j \neq n} \xi_{n j}^{(n)}
\end{array}\right)
$$

\footnotetext{
${ }^{1}$ Supported in part by NSF \#DMS-0449365, School of Statistics, University of Minnesota, 224 Church Street, MN55455, tjiang@stat.umn.edu.

Key Words: random matrix, random graph, dilute graph, Laplacian matrix, normalized Laplacian matrix, spectral distribution, semi-circle law, free convolution.

AMS (2000) subject classifications: 05C80, 05C50, 15A52, 60B10.
} 
The normalized Laplacian matrix is defined by

$$
\mathbf{L}_{n}=\mathbf{I}_{n}-\mathbf{D}_{n}^{-1 / 2} \mathbf{A}_{n} \mathbf{D}_{n}^{-1 / 2}
$$

where $\mathbf{D}_{n}=\left(\sum_{j \neq i}^{n} \xi_{i j}^{(n)}\right)_{1 \leq i \leq n}$ and $\mathbf{D}_{n}^{-1 / 2}=\left(\left(\sum_{j \neq i}^{n} \xi_{i j}^{(n)}\right)^{-1 / 2}\right)_{1 \leq i \leq n}$ with $0^{-1 / 2}:=0$.

The matrix $\boldsymbol{\Delta}_{n}$ is always non-negative definite, and the smallest eigenvalue is zero. The Kirchhoff theorem [30] establishes the relationship between the number of the spanning trees of the graph and the eigenvalues of $\boldsymbol{\Delta}_{n}$. The matrix $\mathbf{L}_{n}$ is a different version of $\boldsymbol{\Delta}_{n}$. It looks a bit complicated, however, the spectrum of $\mathbf{L}_{n}$ is related to the graph discrepancy (or the graph invariant), and the second smallest eigenvalue of $\mathbf{L}_{n}$ relates to the Cheeger constant and the rate convergence of random walks on the graph, see, e.g., $[8,9]$.

The spectral properties of the graph-relevant matrices such as $\mathbf{A}_{n}, \boldsymbol{\Delta}_{n}$ and $\mathbf{L}_{n}$ have applications in chemistry [4], where eigenvalues were connected to the stability of molecules. They are also studied and used in theoretical physics and quantum mechanics, e.g., [21, 22, 23, 24, 25, 36, 37].

For graph $G\left(n, p_{n}\right)$ there are two major asymptotic regimes: $n p_{n} \rightarrow \infty$ and $n p_{n} \rightarrow c \in(0, \infty)$. The former corresponds to the dilute model, and the latter corresponds to the sparse model. Of course, the dilute model consists of two important cases: $p_{n} \equiv p$ and $p_{n} \rightarrow 0$ with $n p_{n} \rightarrow \infty$.

There are several work on $\boldsymbol{\Delta}_{n}$. Bauer and Golinelli [3] simulate the eigenvalues for the ErdösRényi random graph with $p_{n} \equiv p$. Assuming the entries $\left\{\xi_{i j}^{(n)} \equiv \xi_{i j} ; 1 \leq i<j \leq n<\infty\right\}$ in $\boldsymbol{\Delta}_{n}$ in (1.2) are independent and identically distributed random variables with mean zero, Bryc, Dembo and Jiang [6] prove that the empirical distribution of the eigenvalues of $\boldsymbol{\Delta}_{n}$ converges to the free convolution $\gamma_{M}$ of the semi-circular law and $N(0,1)$. This results does not apply to the Erdös-Rényi random graph directly because the means of $\xi_{i j}$ 's in the graph are not zero. A result from Ding and Jiang [15] complements their result in [6] by showing that, under a general framework including the Erdös-Rényi random graph $G\left(n, p_{n}\right)$ with $p_{n} \equiv p$, the corresponding empirical distribution converges to $\gamma_{M}$. When $\xi_{i j}^{(n)}$ 's in (1.2) are deterministic, some results are given for the normalized Laplacian matrix $\mathbf{L}_{n}$ in [8].

In this paper, for the Erdös-Rényi random graph $G\left(n, p_{n}\right)$ with $n p_{n} \rightarrow \infty$, we study the empirical distributions of the eigenvalues of $\boldsymbol{\Delta}_{n}$ and $\mathbf{L}_{n}$. Before stating the main results, we review some notation.

Given an $n \times n$ symmetric matrix $\mathbf{M}$, let $\lambda_{1} \geq \lambda_{2} \geq \cdots \geq \lambda_{n}$ be the eigenvalues of $\mathbf{M}$, we sometimes also write this as $\lambda_{1}(\mathbf{M}) \geq \lambda_{2}(\mathbf{M}) \geq \cdots \geq \lambda_{n}(\mathbf{M})$ for clarity. The notation $\lambda_{\max }=$ $\lambda_{\max }(\mathbf{M}), \lambda_{\min }=\lambda_{\min }(\mathbf{M})$ stand for the largest eigenvalue and the smallest eigenvalue of $\mathbf{M}$, respectively. The following is the situation we will consider in this paper.

Let $\left\{\xi_{i j}^{(n)} ; 1 \leq i<j<\infty\right\}$ be defined on the same probability space with $\xi_{i i}^{(n)}=0$ and $\xi_{i j}^{(n)}=\xi_{j i}^{(n)}$ for all $1 \leq i, j \leq n<\infty$. For each $n \geq 2$, assume $\left\{\xi_{i j}^{(n)} ; 1 \leq i<j \leq n\right\}$ are i.i.d. with $P\left(\xi_{12}^{(n)}=1\right)=1-P\left(\xi_{12}^{(n)}=0\right)=p_{n} \in(0,1)$.

For an $n \times n$ matrix $\mathbf{M}$, we use $\|\mathbf{M}\|=\sup _{\mathbf{x} \in \mathbb{R}^{n}:\|\mathbf{x}\|=1}\|\mathbf{M x}\|$ to denote its spectral norm, where $\|\mathbf{x}\|=\sqrt{x_{1}^{2}+\cdots+x_{n}^{2}}$ for $\mathbf{x}=\left(x_{1}, \cdots, x_{n}\right)^{\prime} \in \mathbb{R}^{n}$. The following are the main results of this paper. 
THEOREM 1 Suppose (1.4) holds with $\alpha_{n}:=\left(n p_{n}\left(1-p_{n}\right)\right)^{1 / 2} \rightarrow \infty$ as $n \rightarrow \infty$, then, almost surely,

$$
F_{n}(x):=\frac{1}{n} \sum_{i=1}^{n} I\left\{\frac{\lambda_{i}\left(\boldsymbol{\Delta}_{n}\right)-n p_{n}}{\sqrt{n p_{n}\left(1-p_{n}\right)}} \leq x\right\}, \quad x \in \mathbb{R},
$$

converges weakly to the free convolution $\gamma_{M}$ of the semi-circle law and $N(0,1)$.

It is known from [6] that the measure $\gamma_{M}$ is a non-random symmetric probability measure with smooth bounded density, doesn't depend on $p_{n}$ 's and has an unbounded support. Theorem 1.3 from [6] and Theorem 2 from [15] deal with similar problems by assuming $\xi_{i j}^{(n)}$ 's in (1.2) being arbitrary random variables of certain finite moments. With truncations on $\xi_{i j}^{(n)}$ 's, the proofs of the two theorems from $[6,15]$ are essentially reduced to the case that $\xi_{i j}^{(n)}$ 's are bounded. Here the situation is different: we will need to work on $\left(\xi_{i j}^{(n)}-p_{n}\right) / \sqrt{p_{n}}$ 's, which are unbounded since $p_{n} \rightarrow 0$ in one of the dilute cases mentioned earlier. The truncation method used in $[6,15]$ does not apply here simply because the current random variables just take two values. In fact, more involved analysis and combinatorics are used in the proof of Theorem 1.

If $p_{n}$ in Theorem 1 is equal to a constant, we get the following result.

COROLlaRY 1.1 Suppose (1.4) holds with $p_{n} \equiv p \in(0,1)$ for all $n \geq 2$. Then, almost surely,

$$
F_{n}(x):=\frac{1}{n} \sum_{i=1}^{n} I\left\{\frac{\lambda_{i}\left(\boldsymbol{\Delta}_{n}\right)-n p}{\sqrt{n p(1-p)}} \leq x\right\}, \quad x \in \mathbb{R},
$$

converges weakly to the free convolution $\gamma_{M}$ of the semi-circle law and $N(0,1)$.

Take $p_{n}$ in Theorem 1 to be a special dilute case: $p_{n} \rightarrow 0$ with $n p_{n} \rightarrow+\infty$ as $n \rightarrow \infty$. Note that, under this condition, $\sqrt{n p_{n}\left(1-p_{n}\right)} \sim \sqrt{n p_{n}}$ as $n \rightarrow \infty$, we then easily have the following.

COROLlARY 1.2 Suppose (1.4) holds with $p_{n} \rightarrow 0$ and $n p_{n} \rightarrow \infty$ as $n \rightarrow \infty$, then, almost surely,

$$
F_{n}(x):=\frac{1}{n} \sum_{i=1}^{n} I\left\{\frac{\lambda_{i}\left(\boldsymbol{\Delta}_{n}\right)-n p_{n}}{\sqrt{n p_{n}}} \leq x\right\}, \quad x \in \mathbb{R},
$$

converges weakly to the free convolution $\gamma_{M}$ of the semi-circle law and $N(0,1)$.

Now we study the normalized Laplacian matrix $\mathbf{L}_{n}$ in (1.3).

THEOREM 2 Suppose (1.4) holds with $\sup \left\{p_{n} ; n \geq 2\right\}<1$. If $n p_{n} / \log n \rightarrow \infty$ as $n \rightarrow \infty$, then, with probability one,

$$
F_{n}(x):=\frac{1}{n} \sum_{i=1}^{n} I\left(\sqrt{\frac{n p_{n}}{1-p_{n}}}\left(1-\lambda_{i}\left(\mathbf{L}_{n}\right)\right) \leq x\right), \quad x \in \mathbb{R},
$$

converges weakly to the semi-circle law with density $\frac{1}{2 \pi} \sqrt{4-x^{2}} I(|x| \leq 2)$. 
Fan, $\mathrm{Lu}$ and $\mathrm{Vu}[11]$ derive a result for a weak form of $\mathbf{L}_{n}$ when the graphs are of the given expected degrees, of which the Erdös-Rényi model is a special case. The three authors prove that the the empirical distribution of the eigenvalues of $\mathbf{I}_{n}-\tilde{\mathbf{D}}_{n}^{-1 / 2} \mathbf{A}_{n} \tilde{\mathbf{D}}_{n}^{-1 / 2}$ converges to the semi-circle law in probability, where $\tilde{\mathbf{D}}_{n}$ is the mathematical expectation of $\mathbf{D}_{n}$.

When $p_{n}$ does not depend on $n$, the above theorem obviously implies the following.

COROLlaRY 1.3 Suppose (1.4) holds with $p_{n} \equiv p \in(0,1)$ for all $n \geq 2$. Then, with probability one,

$$
F_{n}(x):=\frac{1}{n} \sum_{i=1}^{n} I\left(\sqrt{\frac{n p}{1-p}}\left(1-\lambda_{i}\left(\mathbf{L}_{n}\right)\right) \leq x\right), \quad x \in \mathbb{R}
$$

converges weakly to the semi-circle law with density $\frac{1}{2 \pi} \sqrt{4-x^{2}} I(|x| \leq 2)$.

If $p_{n} \rightarrow 0$, then $\sqrt{\frac{n p_{n}}{1-p_{n}}} \sim \sqrt{n p_{n}}$ as $n \rightarrow \infty$. From Theorem 2 we immediately obtain a limiting result for the dilute Erdös-Rényi random graph.

COROllaRY 1.4 Suppose (1.4) holds with $p_{n} \rightarrow 0$ and $n p_{n} / \log n \rightarrow \infty$ as $n \rightarrow \infty$, then, almost surely,

$$
F_{n}(x):=\frac{1}{n} \sum_{i=1}^{n} I\left(\sqrt{n p_{n}}\left(1-\lambda_{i}\left(\mathbf{L}_{n}\right)\right) \leq x\right), \quad x \in \mathbb{R}
$$

converges weakly to the semi-circle law with density $\frac{1}{2 \pi} \sqrt{4-x^{2}} I(|x| \leq 2)$.

In the general context of random graphs, the limits of the empirical distributions of the eigenvalues of $\mathbf{A}_{n}, \boldsymbol{\Delta}_{n}$ and $\mathbf{L}_{n}$ in (1.1), (1.2) and (1.3) respectively are related to the three distributions: the Wigner's semi-circle law, the free convolution of the semi-circle law and $N(0,1)$, and the KestenMcKay law. The last is the limit of the empirical distributions of the eigenvalues of $\mathbf{A}_{n}$ for the random $d$-regular graphs, see [33].

REMARK 1.1 The above theorems study the limiting spectral distributions of $\boldsymbol{\Delta}_{n}$ and $\mathbf{L}_{n}$ for the dilute case. There are some simulations and theoretical work on $\mathbf{A}_{n}$ and $\boldsymbol{\Delta}_{n}$ for the sparse case in [3, 26, 29, 32, 36, 37, 38], that is, $p_{n}=c / n$ for all $n \geq 2$ and $c$ is a constant. It seems that the limits of the spectral distributions of $\mathbf{A}_{n}$ and $\boldsymbol{\Delta}_{n}$ are still not identified.

REMARK 1.2 Theorems 1 and 2 are derived based on the Erdös-Rényi model. The methods of the proofs could be carried to more complex models, for instance, the random geometric graphs [34], the weighted dilute random matrices [28], the power law random graphs, and the random graphs with given expected degrees [9].

REMARK 1.3 There are a couple of books on the study of the spectral properties of the matrices generated from graphs, see, e.g., [1, 8, 9, 12, 13, 35] for reference.

The rest of the paper is organized as follows: the proof of Theorem 1 is given in Section 2; the proof of Theorem 2 is given in Section 3. 


\section{Proof of Theorem 1}

Given $n \geq 2$, let $\Gamma_{n}=\{(i, j) ; 1 \leq j<i \leq n\}$ be a graph. Any element $(i, j) \in \Gamma_{n}$ is called a vertex, and $i$ and $j$ are called indices. We say vertices $a=\left(i_{1}, j_{1}\right)$ and $b=\left(i_{2}, j_{2}\right)$ form an edge and denote it by $a \sim b$, if one of $i_{1}$ and $j_{1}$ is identical to one of $i_{2}$ and $j_{2}$. For convenience of notation, from now on, we write $a=\left(a^{+}, a^{-}\right)$for any $a \in \Gamma_{n}$. Of course, $a^{+}>a^{-}$. Given $a, b \in \Gamma_{n}$, define an $n \times n$ matrix

$$
\mathbf{Q}_{a, b}[i, j]= \begin{cases}-1, & \text { if } i=a^{+}, j=b^{+}, \text {or } i=a^{-}, j=b^{-} \\ 1, & \text { if } i=a^{+}, j=b^{-}, \text {or } i=a^{-}, j=b^{+} \\ 0, & \text { otherwise. }\end{cases}
$$

Recall (1.4). Set

$$
\eta_{i j}^{(n)}=\frac{\xi_{i j}^{(n)}-p_{n}}{\sqrt{p_{n}\left(1-p_{n}\right)}}
$$

for all $1 \leq i \neq j \leq n$ and $n \geq 2$, and

$$
\mathbf{\Psi}_{n}=\left(\begin{array}{cccc}
\sum_{j \neq 1} \eta_{1 j}^{(n)} & -\eta_{12}^{(n)} & \ldots & -\eta_{1 n}^{(n)} \\
-\eta_{21}^{(n)} & \sum_{j \neq 2} \eta_{2 j}^{(n)} & \ldots & -\eta_{2 n}^{(n)} \\
\vdots & \vdots & \vdots & \vdots \\
-\eta_{n 1}^{(n)} & -\eta_{n 2}^{(n)} & \cdots & \sum_{j \neq n} \eta_{n j}^{(n)}
\end{array}\right) .
$$

With this and the above notation, we rewrite $\boldsymbol{\Psi}_{n}$ as follows.

$$
-\mathbf{\Psi}_{n}=\sum_{a \in \Gamma_{n}} \eta_{a}^{(n)} \mathbf{Q}_{a, a}
$$

where $\eta_{a}^{(n)}=\eta_{a^{+} a^{-}}^{(n)}$ for $a=\left(a^{+}, a^{-}\right) \in \Gamma_{n}$. Let $t_{a, b}=\operatorname{tr}\left(\mathbf{Q}_{a, b}\right)$. We summarize some facts from [6] in the following lemma.

LEMMA 2.1 Let $a, b \in \Gamma_{n}$. The following assertions hold:

(i) $t_{a, b}=t_{b, a}$.

(ii) $t_{a, b}= \begin{cases}-2, & \text { if } a=b ; \\ -1, & \text { if } a \neq b \text { and } a^{-}=b^{-} \text {or } a^{+}=b^{+} \\ 1, & \text { if } a \neq b \text { and } a^{-}=b^{+} \text {or } a^{+}=b^{-} \\ 0, & \text { otherwise. }\end{cases}$

(iii) $\mathbf{Q}_{a, b} \times \mathbf{Q}_{c, d}=t_{b, c} \mathbf{Q}_{a, d}$. Therefore, $\operatorname{tr}\left(\mathbf{Q}_{a_{1}, a_{1}} \times \mathbf{Q}_{a_{2}, a_{2}} \times \cdots \times \mathbf{Q}_{a_{r}, a_{r}}\right)=\prod_{j=1}^{r} t_{a_{j}, a_{j+1}}$, where $a_{1}, \cdots, a_{r} \in \Gamma_{n}$, and $a_{r+1}=a_{1}$.

Given $n \geq 2$, we call $\pi=\left(a_{1}, \cdots, a_{r}\right)$ a circuit of length $r$ in $\Gamma_{n}$ if $a_{i} \in \Gamma_{n}$ for $1 \leq i \leq n$ and $a_{1} \sim \cdots \sim a_{r} \sim a_{1}$. For such a circuit, set

$$
\eta_{\pi}^{(n)}=\left(\prod_{j=1}^{r} t_{a_{j}, a_{j+1}}\right) \prod_{j=1}^{r} \eta_{a_{j}}^{(n)}
$$


with $a_{r+1}=a_{1}$. From (2.3) and (iii) of Lemma 2.1, we know

$$
\operatorname{tr}\left(\mathbf{\Psi}_{n}^{r}\right)=(-1)^{r} \sum_{\pi} \eta_{\pi}^{(n)} \text { and } E \operatorname{tr}\left(\mathbf{\Psi}_{n}^{r}\right)=(-1)^{r} \sum_{\pi} E \eta_{\pi}^{(n)}
$$

where the sum is taken over all circuits of length $r$ in $\Gamma_{n}$.

DEFINITION 2.1 We say that a circuit $\pi=\left(a_{1} \sim \cdots \sim a_{r} \sim a_{1}\right)$ of length $r$ in $\Gamma_{n}$ is vertexmatched if for each $i=1, \cdots, r$ there exists some $j \neq i$ such that $a_{j}=a_{i}$; we say $\pi$ has a match of order 3 if some value is repeated at least three times among $\left\{a_{j}, j=1, \cdots, r\right\}$.

Clearly, by independence, the only possible non-zero terms in (2.5) come from vertex-matched circuits. For $x \geq 0$, denote by $\lfloor x\rfloor$ the integer part of $x$. The following two lemmas will be used later.

LemMa 2.2 (Proposition 4.10 from [6]). Fix $r \in \mathbb{N}$. Let $N$ denote the number of vertex-matched circuits in $\Gamma_{n}$ with vertices having at least one match of order 3. Then $N=O\left(n^{\lfloor(r+1) / 2\rfloor}\right)$ as $n \rightarrow \infty$.

Recall $\Gamma_{n}=\{(i, j) ; 1 \leq j<i \leq n\}$ for $n \geq 2$. Let $m, r, s_{1}, \cdots, s_{m}$ be positive integers with $r \geq m$ and $s_{1}+\cdots+s_{m}=r$. Define $A_{m, n, r}$ by

$\left\{a_{1} \sim a_{2} \sim \cdots \sim a_{r} \sim a_{1}: a_{i} \in \Gamma_{n}\right.$ for $1 \leq i \leq n$; there exist pairwise different $a_{1}^{\prime}, \cdots, a_{m}^{\prime} \in$

$\left\{a_{1}, \cdots, a_{r}\right\}$ such that $a_{i}^{\prime}$ appears $s_{i}$ times in the sequence $a_{1} a_{2} \cdots a_{r}$ for $\left.1 \leq i \leq m\right\}$.

LEMMA 2.3 Let $m, r, s_{1}, \cdots, s_{m}$ be positive integers with $r \geq m$ and $s_{1}+\cdots+s_{m}=r$. Then, there exists a constant $C_{r}$ depending only on $r$ such that $\left|A_{m, n, r}\right| \leq C_{r} \cdot n^{m+1}$ for all $n \geq 2$.

Proof. First, denote by $B$ the total number of ways to assign $m$ different items, say, $\omega_{1}, \cdots, \omega_{m}$, into a sequence of length $r$ such that $\omega_{i}$ appears exactly $s_{i}$ times for each $1 \leq i \leq m$. Then $B=\left(\begin{array}{c}r \\ s_{1}, \cdots, s_{l}\end{array}\right) \leq r !$

Given one of such assignment, recalling (2.6), the number of choices of $a_{1}=\left(a_{1}^{+}, a_{1}^{-}\right) \in \Gamma_{n}$ is at most $n^{2}$. Then, each time a new vertex $a_{i}^{\prime}=\left(a_{i}^{+}, a_{i}^{-}\right)$appears in the circuit sequence, one index from $\left\{a_{i}^{+}, a_{i}^{-}\right\}$is free, while the other is identical to one of $\left\{a^{+}, a^{-}\right\}$'s which appear in the circuit sequence earlier than $a_{i}^{\prime}$. Thus, the total number of the choices of $a_{i}^{\prime}$ is bounded by $2^{r-1} n+2^{r-1} n=2^{r} n$. It follows that the total number of the circuits corresponding to the assignment is no more than $n^{2} \cdot\left(2^{r} n\right)^{m-1} \leq 2^{r^{2}} n^{m+1}$. Take $C_{r}=2^{r^{2}} r$ !. The conclusion then follows by combining the two steps.

Recall vertex $a=\left(a^{+}, a^{-}\right) \in \Gamma_{n}$ has two end points $a^{+}$and $a^{-}$.

DEFINITION 2.2 Fix integer $n \geq 2$. Let $\pi_{1}, \pi_{2}, \pi_{3}, \pi_{4}$ be four circuits of length $r$ in $\Gamma_{n}$, we say they are matched if each vertex of any one of these circuits is either self-matched, that is, there is another vertex of the same circuit with the same end points, or is cross-matched, that is, there is a vertex of the other circuit with the same end points (or both).

Given $l \in \mathbb{N}$, let $Q_{l}$ be the number of the above matched quadruples satisfying: (a) for any $i=1,2,3,4$, there is $j \neq i$ such that $\pi_{i}$ and $\pi_{j}$ share a common vertex; (b) the total number of different vertices in the four circuits is $l$. 
LEMMA 2.4 For fixed $l, r \in \mathbb{N}$ with $1 \leq l \leq 2 r$, there exists a constant $C_{r}$ not depending on $n$, such that $Q_{l} \leq C_{r} n^{l+2}$ for all $n \geq 2$.

Proof. Write

$$
\begin{aligned}
& \pi_{1}=a_{1} \sim a_{2} \sim \cdots \sim a_{r} \sim a_{1} ; \\
& \pi_{2}=b_{1} \sim b_{2} \sim \cdots \sim b_{r} \sim b_{1} ; \\
& \pi_{3}=c_{1} \sim c_{2} \sim \cdots \sim c_{r} \sim c_{1} ; \\
& \pi_{4}=d_{1} \sim d_{2} \sim \cdots \sim d_{r} \sim d_{1} .
\end{aligned}
$$

For $i \neq j$, denote by $\pi_{i} \leftrightarrow \pi_{j}$ if $\pi_{i}$ and $\pi_{j}$ have at least one crossed match. Up to the permutations of $\pi_{1}, \pi_{2}, \pi_{3}, \pi_{4}$, all matched quadruples satisfying (a) above the statement of the lemma can be divided into three cases: (i) $\pi_{1} \leftrightarrow \pi_{2}$ and $\pi_{3} \leftrightarrow \pi_{4}$; (ii) $\pi_{1} \leftrightarrow \pi_{2}, \pi_{1} \leftrightarrow \pi_{3}$ and $\pi_{2} \leftrightarrow \pi_{4}$; (iii) $\pi_{1} \leftrightarrow \pi_{2}, \pi_{1} \leftrightarrow \pi_{3}$ and $\pi_{1} \leftrightarrow \pi_{4}$. (By "permutations" we mean that the circuits $\pi_{1}, \pi_{2}, \pi_{3}, \pi_{4}$ in (i), (ii) and (iii) can be $\pi_{i_{1}}, \pi_{i_{2}}, \pi_{i_{3}}, \pi_{i_{4}}$ for any permutation $\left\{i_{1}, i_{2}, i_{3}, i_{4}\right\}$ of $\{1,2,3,4\}$ ). Thus, to prove the lemma, it suffices to show that the total number of the circuits in each of the three cases are no more than $\mathrm{Cn}^{l+2}$ for some constant $C$ not depending on $n$. To do so, since each of the four circuits has length $r$, it is enough to show the total number of the vertices in the four circuits is bounded by $C n^{l+2}$ with $C$ not depending on $n$.

Case (i): $\pi_{1} \leftrightarrow \pi_{2}$ and $\pi_{3} \leftrightarrow \pi_{4}$. Suppose there are $l_{1}$ different vertices among circuits $\pi_{1}$ and $\pi_{2}$, then there are $l_{2}=l-l_{1} \geq 0$ different vertices in $\pi_{3}$ and $\pi_{4}$ that do not appear in $\pi_{1}$ and $\pi_{2}$.

Since $\pi_{1} \leftrightarrow \pi_{2}$, recalling (2.7), say, $\left(a_{1}^{+}, a_{1}^{-}\right)=a_{1} \sim b_{1}$. Then, there are at most $n(n-1) / 2$ ways to assign $\{1, \cdots, n\}$ to $a_{1}^{+}$and $a_{1}^{-}$with $a_{1}^{+}>a_{1}^{-}$. Now assign such numbers to other $l_{1}-1$ vertices, there are at most $2 n$ assignment for each of a new vertex since two distinct neighbors are connected. So the total number of vertices in $\pi_{1}$ and $\pi_{2}$ is no more than $n^{2} \cdot(2 n)^{l_{1}-1} \leq 2^{l_{1}} n^{l_{1}+1}$. Similarly, there are at most $2^{l_{2}} n^{l_{2}+1}$ different vertices in circuits $\pi_{3}$ and $\pi_{4}$. So the total number of vertices in circuits $\pi_{1}, \pi_{2}, \pi_{3}, \pi_{4}$ is bounded by $2^{l_{1}} n^{l_{1}+1} \cdot 2^{l_{2}} n^{l_{2}+1}=2^{l} n^{l+2}$.

Cases (ii) and (iii). The difference between the two cases and case (i) is that the four circuits here are all connected, while $\left(\pi_{1}, \pi_{2}\right)$ and $\left(\pi_{3}, \pi_{4}\right)$ in case (i) may not have a common vertex at all. For $i=1,2,3,4$, let $u_{i}$ be the number of the distinct vertices in $\pi_{i}$ that do not appear in $\pi_{j}$ for $j<i$. Then $u_{1}+u_{2}+u_{3}+u_{4}=l$. We proceed to assign $(i, j)$ 's with $i>j$ to the vertices in $\pi_{1}$ first, then $\pi_{2}, \pi_{3}, \pi_{4}$ consecutively. By the same consideration as that in (i), the total number of possible vertices in $\pi_{1}$ is at most $n^{u_{1}+1}$. Since for any $i=2,3,4$, there exists $j=1,2,3$ such that $\pi_{j} \leftrightarrow \pi_{i}$, then the total number of different vertices in $\pi_{k}$ is at most $(2 n)^{u_{k}}$ for $k=2,3,4$. Therefore, the total number of the distinct vertices in the four circuits is at most $n^{u_{1}+1} \cdot(2 n)^{u_{2}} \cdot(2 n)^{u_{3}} \cdot(2 n)^{u_{4}} \leq 2^{l} n^{l+1}$.

LemMA 2.5 Let $m, n \in \mathbb{N}$. Let $X_{1}, \cdots, X_{n}$ be non-negative, independent random variables. Set $g\left(a_{1}, \cdots, a_{n}\right)=E\left(X_{1}^{a_{1}} \cdots X_{n}^{a_{n}}\right)<\infty$ for non-negative integers $a_{1}, \cdots, a_{n}$ with $0^{0}=1$. Then

$$
g\left(a_{1}, \cdots, a_{n}\right) \geq \prod_{j=1}^{m} g\left(a_{1 j}, \cdots, a_{n j}\right)
$$


where $\left\{a_{i j} ; 1 \leq i \leq n, 1 \leq j \leq m\right\}$ are nonnegative integers satisfying $\sum_{j=1}^{m} a_{i j}=a_{i}$ for all $1 \leq i \leq n$.

Proof. Given random variable $Y \geq 0$ and non-decreasing functions $f(x)$ and $g(x)$ on $[0, \infty)$. If $E|f(Y)|<\infty, E|g(Y)|<\infty$ and $E|f(Y) g(Y)|<\infty$, then, by the covariance inequality, we have that $E(f(Y) g(Y)) \geq E f(Y) \cdot E g(Y)$. Thus, for non-negative integers $b, b_{1}, \cdots, b_{m}$ with $b=b_{1}+\cdots+b_{m}$, we obtain

$$
E\left(X_{i}^{b}\right) \geq E\left(X_{i}^{b_{1}}\right) \cdot E\left(X_{i}^{b_{2}+\cdots+b_{n}}\right) \geq \cdots \geq \prod_{j=1}^{n} E\left(X_{i}^{b_{j}}\right)
$$

for any $1 \leq i \leq n$. By independence,

$$
g\left(a_{1}, \cdots, a_{n}\right)=\prod_{i=1}^{n} E\left(X_{i}^{a_{i}}\right) \text { and } \prod_{j=1}^{m} g\left(a_{1 j}, \cdots, a_{n j}\right)=\prod_{i=1}^{n} \prod_{j=1}^{m} E\left(X_{i}^{a_{i j}}\right) .
$$

From (2.8), $E\left(X_{i}^{a_{i}}\right) \geq \prod_{j=1}^{m} E\left(X_{i}^{a_{i j}}\right)$ since $\sum_{j=1}^{m} a_{i j}=a_{i}$ for all $i=1,2, \cdots, n$. Then the conclusion follows.

Recall from (2.5) that $n^{-r / 2-1} E \operatorname{tr}\left(\boldsymbol{\Psi}_{n}^{r}\right)=(-1)^{r} n^{-r / 2-1} \sum_{\pi} E \eta_{\pi}^{(n)}$ where the sum is taken over all circuits of length $r$ in $\Gamma_{n}$. We next show that some terms in the sum are negligible.

LEMMA 2.6 Suppose the conditions in Theorem 1 hold. Fix $r \in \mathbb{N}$. Let $W_{n}=n^{-r / 2-1} \sum_{\pi \in \Lambda_{n}} E \eta_{\pi}^{(n)}$, where $\Lambda_{n}$ is the set of vertex matched circuits of order 3 and of length $r$ in $\Gamma_{n}$. Then $W_{n} \rightarrow 0$ as $n \rightarrow \infty$.

Proof. Take a circuit in $\Lambda_{n}: a_{1} \sim a_{2} \sim \cdots \sim a_{r} \sim a_{1}$. Let $a_{1}^{\prime}, \cdots, a_{m}^{\prime} \in\left\{a_{1}, \cdots, a_{r}\right\}$ be pairwise different vertices with $a_{i}^{\prime}$ appearing $s_{i} \geq 2$ times in the sequence $a_{1} a_{2} \cdots a_{r}$ for $1 \leq i \leq m$ with $s_{1}+\cdots+s_{m}=r$ and $\max _{1 \leq i \leq m} s_{i} \geq 3$. Evidently, $m \leq(r-1) / 2$. By $(2.4)$ and independence,

$$
\left|E \eta_{\pi}^{(n)}\right|=\left|\left(\prod_{j=1}^{r} t_{a_{j}, a_{j+1}}\right) \cdot E \prod_{i=1}^{m}\left(\eta_{a_{i}^{\prime}}^{(n)}\right)^{s_{i}}\right| \leq 2^{r} \cdot \prod_{i=1}^{m} E\left(\left|\eta_{a_{i}^{\prime}}^{(n)}\right|^{s_{i}}\right),
$$

where the last inequality is from (ii) of Lemma 2.1. Now, let $Z \sim \operatorname{Ber}\left(p_{n}\right)$ and $q_{n}=1-p_{n}$. Notice $p_{n}^{s_{i}} q_{n}+p_{n} q_{n}^{s_{i}} \leq 2 p_{n} q_{n}$, we obtain from (2.1) that $E\left(\left|\eta_{a_{i}^{\prime}}^{(n)}\right|^{s_{i}}\right)$ is identical to

$$
E\left(\frac{\left|Z-p_{n}\right|}{\sqrt{p_{n} q_{n}}}\right)^{s_{i}}=\frac{p_{n}^{s_{i}} q_{n}+p_{n} q_{n}^{s_{i}}}{\left(p_{n} q_{n}\right)^{s_{i} / 2}} \leq \frac{2}{\left(p_{n} q_{n}\right)^{s_{i} / 2-1}}
$$

Using $s_{1}+\cdots+s_{m}=r$ to see that $\left|E \eta_{\pi}^{(n)}\right| \leq 2^{r} \cdot 2^{m}\left(p_{n} q_{n}\right)^{m-(r / 2)}$. Let $C_{m, r}$ be the number of solutions of the equation $s_{1}+\cdots+s_{m}=r$ with integer $s_{i} \geq 2$ for each $1 \leq i \leq m$. Now we classify the sum $\sum_{\pi \in \Lambda_{n}} E \eta_{\pi}^{(n)}$ into the sums corresponding to $m=1,2, \cdots,\lfloor(r-1) / 2\rfloor$. By Lemma 2.3, the $m$-th sum is bounded by $C_{m, r} \cdot C_{r} n^{m+1} \cdot 2^{m+r}\left(p_{n} q_{n}\right)^{m-(r / 2)}$. It follows that

$$
\begin{aligned}
\left|W_{n}\right| & \leq \frac{1}{n^{r / 2+1}} \sum_{1 \leq m \leq(r-1) / 2} C_{m, r} \cdot C_{r} n^{m+1} \cdot 2^{m+r}\left(p_{n} q_{n}\right)^{m-(r / 2)} \\
& \leq C \sum_{1 \leq m \leq(r-1) / 2}\left(n p_{n} q_{n}\right)^{m-(r / 2)}=O\left(\frac{1}{\sqrt{n p_{n} q_{n}}}\right) \rightarrow 0
\end{aligned}
$$


as $n \rightarrow \infty$ since $n p_{n} q_{n} \rightarrow \infty$ by assumption, where $C$ above is a constant not depending on $n$.

Let $\mathbf{U}_{n}$ be a symmetric matrix of form

$$
\mathbf{U}_{\mathbf{n}}=\left(\begin{array}{cccc}
\sum_{j \neq 1} Y_{1 j} & -Y_{12} & \cdots & -Y_{1 n} \\
-Y_{21} & \sum_{j \neq 2} Y_{2 j} & \cdots & -Y_{2 n} \\
\vdots & \vdots & \vdots & \vdots \\
-Y_{n 1} & -Y_{n 2} & \cdots & \sum_{j \neq n} Y_{n j}
\end{array}\right)
$$

where $\left\{Y_{i j} ; 1 \leq i<j<\infty\right\}$ are i.i.d. standard normal random variables not depending on $n$.

LEMMA 2.7 Suppose the conditions in Theorem 1 hold. Let $\Psi_{n}$ and $\mathbf{U}_{n}$ be as in (2.2) and (2.10) respectively. Then

(i) $\lim _{n \rightarrow \infty} \frac{1}{n^{k+1 / 2}} E \operatorname{tr}\left(\mathbf{\Psi}_{n}^{2 k-1}\right)=0$;

(ii) $\lim _{n \rightarrow \infty} \frac{1}{n^{k+1}}\left(E \operatorname{tr}\left(\mathbf{\Psi}_{n}^{2 k}\right)-E \operatorname{tr}\left(\mathbf{U}_{n}^{2 k}\right)\right)=0$

for any integer $k \geq 1$.

Proof. (i) As in (2.5), $E \operatorname{tr}\left(\mathbf{\Psi}_{n}^{2 k-1}\right)=\sum_{\pi} E \eta_{\pi}^{(n)}$ where the sum is taken over all circuits of length $2 k-1$ in $\Gamma_{n}$ in which each vertex appearing at least twice. Notice $r=2 k-1$ is odd, one vertex has to appear at least three times. The result then follows from Lemma 2.6.

(ii) Recall (2.10). For a circuit $a_{1} \sim \cdots \sim a_{r} \sim a_{1}$ with $a_{i} \in \Gamma_{n}$ for all $1 \leq i \leq r$, similar to notation $\eta_{\pi}^{(n)}$ in (2.4), we define

$$
Y_{\pi}^{(n)}=\left(\prod_{j=1}^{r} t_{a_{j}, a_{j+1}}\right) \prod_{j=1}^{r} Y_{a_{j}} .
$$

Taking $r=2 k$, we have

$$
\begin{aligned}
\left|E \operatorname{tr}\left(\mathbf{\Psi}_{n}^{2 k}\right)-E \operatorname{tr}\left(\mathbf{U}_{n}^{2 k}\right)\right| & =\left|\sum_{\pi}\left(E \eta_{\pi}^{(n)}-E Y_{\pi}^{(n)}\right)\right| \\
& \leq\left|\sum_{\pi \in A_{1}} E \eta_{\pi}^{(n)}\right|+\left|\sum_{\pi \in A_{1}} E Y_{\pi}^{(n)}\right|+\left|\sum_{\pi \in A_{2}}\left(E \eta_{\pi}^{(n)}-E Y_{\pi}^{(n)}\right)\right| \\
& :=I_{1}+I_{2}+I_{3},
\end{aligned}
$$

where $A_{1}$ denotes the set of the vertex-matched circuits with match of order 3 , and $A_{2}$ denotes the set of the vertex-matched circuits in $\Gamma_{n}$ such that there are exact $k$ distinct matches. Now we analyze the three terms one by one.

By Lemma 2.6, $I_{1}=o\left(n^{k+1}\right)$ as $n \rightarrow \infty$. Observe that each vertex of any circuit in $A_{2}$ matches exactly two times. From the independence assumption and that $E\left|\eta_{i j}^{(n)}\right|^{2}=E\left|Y_{i j}\right|^{2}=1$ for all $1 \leq i<j \leq n$ and $n \geq 2$, we know $E \eta_{\pi}^{(n)}=E Y_{\pi}^{(n)}=1$ for all $\pi \in A_{2}$. This gives $I_{3}=0$. Now we turn to estimate $I_{2}$.

Recalling (2.11), for $\pi \in A_{1}, \prod_{j=1}^{2 k} Y_{a_{j}}$ has the form of $\prod_{s=1}^{w} V_{s}^{l_{s}}$, where $V_{1}, \cdots, V_{w}$ are i.i.d. $N(0,1)$-distributed random variables, and $l_{1} \geq 2, \cdots, l_{w} \geq 2$ with $\sum_{s=1}^{w} l_{s}=2 k$. Thus, by the Hölder 
inequality, $E\left|\prod_{j=1}^{2 k} Y_{a_{j}}\right| \leq E\left(|N(0,1)|^{2 k}\right)$. From (ii) of Lemma 2.1, $\left|E Y_{\pi}^{(n)}\right| \leq 2^{2 k} E\left(|N(0,1)|^{2 k}\right)$. It follows that $I_{2} \leq 2^{2 k} E\left(|N(0,1)|^{2 k}\right) \cdot\left|A_{1}\right|=O\left(n^{k}\right)$ as $n \rightarrow \infty$ by Lemma 2.2 .

In summary, $I_{1}+I_{2}+I_{3}=o\left(n^{k+1}\right)$ as $n \rightarrow \infty$, which together with (2.12) concludes (ii).

LEMMA 2.8 Suppose the conditions in Theorem 1 hold. Let $\mathbf{\Psi}_{n}$ be as in (2.2). Then

$$
\lim _{n \rightarrow \infty} \frac{1}{n^{r / 2+1}}\left(\operatorname{tr}\left(\mathbf{\Psi}_{n}^{r}\right)-\operatorname{Etr}\left(\mathbf{\Psi}_{n}^{r}\right)\right)=0 \text { a.s. }
$$

for any integer $r \geq 1$.

Proof. By (2.4) and (2.5),

$$
\operatorname{tr}\left(\mathbf{\Psi}_{n}^{r}\right)-\operatorname{Etr}\left(\mathbf{\Psi}_{n}^{r}\right)=(-1)^{r} \sum_{\pi}\left(\eta_{\pi}^{(n)}-E \eta_{\pi}^{(n)}\right)
$$

where the sum is taken over all circuits of length $r$ in $\Gamma_{n}$, and

$$
\eta_{\pi}^{(n)}=\prod_{j=1}^{r} t_{a_{j}, a_{j+1}} \prod_{j=1}^{r} \eta_{a_{j}}^{(n)}
$$

for $\pi=\left(a_{1}, \cdots, a_{r}\right)$. We claim that, to prove the lemma, it is enough to show

$$
E\left|\operatorname{tr}\left(\mathbf{\Psi}_{n}^{r}\right)-\operatorname{Etr}\left(\mathbf{\Psi}_{n}^{r}\right)\right|^{4}=O\left(n^{2 r+2}\right)
$$

as $n \rightarrow \infty$. In fact, if this holds, by the Markov inequality,

$$
P\left(\frac{1}{n^{r / 2+1}}\left|\operatorname{tr}\left(\mathbf{\Psi}_{n}^{r}\right)-\operatorname{Etr}\left(\mathbf{\Psi}_{n}^{r}\right)\right| \geq \epsilon\right) \leq \frac{E\left|\operatorname{tr}\left(\mathbf{\Psi}_{n}^{r}\right)-E \operatorname{tr}\left(\mathbf{\Psi}_{n}^{r}\right)\right|^{4}}{n^{2 r+4} \epsilon^{4}}=O\left(\frac{1}{n^{2}}\right)
$$

as $n \rightarrow \infty$ for any $\epsilon>0$. Thus, the sum of the left hand side of the above over all $n \geq 2$ is finite, the Borel-Cantelli lemma then gives the desired conclusion. Now,

$$
\begin{aligned}
& E\left|\operatorname{tr}\left(\mathbf{\Psi}_{n}^{r}\right)-E \operatorname{tr}\left(\mathbf{\Psi}_{n}^{r}\right)\right|^{4} \\
= & \sum_{\pi_{1}, \pi_{2}, \pi_{3}, \pi_{4}} E\left\{\left(\eta_{\pi_{1}}^{(n)}-E \eta_{\pi_{1}}^{(n)}\right)\left(\eta_{\pi_{2}}^{(n)}-E \eta_{\pi_{2}}^{(n)}\right)\left(\eta_{\pi_{3}}^{(n)}-E \eta_{\pi_{3}}^{(n)}\right)\left(\eta_{\pi_{4}}^{(n)}-E \eta_{\pi_{4}}^{(n)}\right)\right\}
\end{aligned}
$$

where the sum is taken over all circuits $\pi_{j}, j=1,2,3,4$ of length $r$ each. For every $n \geq 2$, with random variables $\eta_{a}^{(n)}$ 's independent and of mean zero (recalling (2.1)), if a circuit $\pi_{k}$ has a vertex that is not matched with any other vertex in the four circuits, then $E \eta_{\pi_{k}}^{(n)}=0$ and

$$
E \prod_{j=1}^{4}\left(\eta_{\pi_{j}}^{(n)}-E \eta_{\pi_{j}}^{(n)}\right)=E\left\{\eta_{\pi_{k}}^{(n)} \cdot \prod_{j \neq k}\left(\eta_{\pi_{j}}^{(n)}-E \eta_{\pi_{j}}^{(n)}\right)\right\}=0 .
$$

Further, if one of the circuits, say $\pi_{1}$, is only self-matched, i.e., has no cross-matched vertex, then obviously

$$
E \prod_{j=1}^{4}\left(\eta_{\pi_{j}}^{(n)}-E \eta_{\pi_{j}}^{(n)}\right)=E\left(\eta_{\pi_{1}}^{(n)}-E \eta_{\pi_{1}}^{(n)}\right) \cdot E \prod_{j=2}^{4}\left(\eta_{\pi_{j}}^{(n)}-E \eta_{\pi_{j}}^{(n)}\right)=0
$$


Therefore, it suffices to take the sum in (2.14) over all matched quadruples of circuits on $\pi_{1}, \pi_{2}, \pi_{3}, \pi_{4}$ of length $r$ (see Definition 2.2) with the property: for any $i=1,2,3,4$, there is $j \neq i$ such that $\pi_{i}$ and $\pi_{j}$ share the same vertex. Recalling the definition of $Q_{l}$ in Lemma 2.4, since the quadruples are matched, we know $1 \leq l \leq 2 r$, and by this lemma, $\left|Q_{l}\right| \leq C_{r} n^{l+2}$ for all $1 \leq l \leq 2 r$, where $C_{r}$ is a constant depending on $r$ only. Thus, by (2.14),

$$
E\left|\operatorname{tr}\left(\mathbf{\Psi}_{n}^{r}\right)-\operatorname{Etr}\left(\mathbf{\Psi}_{n}^{r}\right)\right|^{4} \leq \sum_{l=1}^{2 r} \sum_{\left(\pi_{1}, \pi_{2}, \pi_{3}, \pi_{4}\right) \in Q_{l}}\left|h\left(\pi_{1}, \pi_{2}, \pi_{3}, \pi_{4}\right)\right|,
$$

where $h\left(\pi_{1}, \pi_{2}, \pi_{3}, \pi_{4}\right)=E \prod_{j=1}^{4}\left(\eta_{\pi_{j}}^{(n)}-E \eta_{\pi_{j}}^{(n)}\right)$. Expand the expectation, there are 16 terms, and the absolute value of each is bounded by $E \prod_{j=1}^{4}\left|\eta_{\pi_{j}}^{(n)}\right|$ by Lemma 2.5. Thus, by (2.15),

$$
E\left|\operatorname{tr}\left(\mathbf{\Psi}_{n}^{r}\right)-\operatorname{Etr}\left(\mathbf{\Psi}_{n}^{r}\right)\right|^{4} \leq 16 C_{r} \sum_{l=1}^{2 r} n^{l+2} \max _{\left(\pi_{1}, \pi_{2}, \pi_{3}, \pi_{4}\right) \in Q_{l}}\left\{E \prod_{j=1}^{4}\left|\eta_{\pi_{j}}^{(n)}\right|\right\} .
$$

Given $l \geq 1$, let $Z$ be a $\operatorname{Ber}\left(p_{n}\right)$-distributed random variable. Then, for each $\left(\pi_{1}, \pi_{2}, \pi_{3}, \pi_{4}\right) \in Q_{l}$, by independence,

$$
E \prod_{j=1}^{4}\left|\eta_{\pi_{j}}^{(n)}\right| \leq \max \prod_{i=1}^{l} E\left(\frac{\left|Z-p_{n}\right|}{\sqrt{p_{n} q_{n}}}\right)^{m_{i}}
$$

where the maximum is over all $m_{1} \geq 1, \cdots, m_{l} \geq 1$ subject to the constraint $m_{1}+\cdots+m_{l}=4 r$. By (2.9), the above is controlled by

$$
\prod_{i=1}^{l} E\left(\frac{\left|Z-p_{n}\right|}{\sqrt{p_{n} q_{n}}}\right)^{m_{i}} \leq \prod_{i=1}^{l} \frac{2}{\left(p_{n} q_{n}\right)^{m_{i} / 2-1}}=\frac{2^{l}}{\left(p_{n} q_{n}\right)^{2 r-l}} .
$$

It follows from (2.16) and (2.17) that

$$
\begin{aligned}
E\left|\operatorname{tr}\left(\mathbf{\Psi}_{n}^{r}\right)-E \operatorname{tr}\left(\mathbf{\Psi}_{n}^{r}\right)\right|^{4} & \leq 16 C_{r} \sum_{l=1}^{2 r} \frac{2^{l} n^{l+2}}{\left(p_{n} q_{n}\right)^{2 r-l}} \\
& \leq\left(16 C_{r} 2^{2 r}\right) n^{2 r+2} \sum_{l=1}^{2 r} \frac{1}{\left(n p_{n} q_{n}\right)^{2 r-l}} \\
& =O\left(n^{2 r+2}\right)
\end{aligned}
$$

as $n \rightarrow \infty$ since $n p_{n} q_{n} \rightarrow \infty$ by assumption. This gives (2.13).

For an $n \times n$ symmetric matrix $\mathbf{M}$, let $F^{\mathbf{M}}$ be the empirical distribution of the eigenvalues of $\mathbf{M}$.

LEMMA 2.9 Suppose $\alpha_{n}:=\left(n p_{n}\left(1-p_{n}\right)\right)^{1 / 2} \rightarrow \infty$ as $n \rightarrow \infty$. Let $\boldsymbol{\Psi}_{n}$ be as in (2.2). Then, as $n \rightarrow \infty$, with probability one, $F^{\boldsymbol{\Psi}_{n} / \sqrt{n}}$ converges weakly to the free convolution $\gamma_{M}$ of the semi-circle law and $N(0,1)$. 
Proof. Proposition A.3 from [6] says that $\gamma_{M}$ is a symmetric distribution and uniquely determined by its moments. Thus, to prove the theorem, it is enough to show that

$$
\begin{aligned}
\frac{1}{n^{r / 2+1}} \operatorname{tr}\left(\mathbf{\Psi}_{n}^{r}\right) & =\frac{1}{n} \operatorname{tr}\left(n^{-1 / 2} \mathbf{\Psi}_{n}\right)^{r}=\int x^{r} d F^{n^{-1 / 2} \mathbf{\Psi}_{n}} \\
& \rightarrow \int x^{r} d \gamma_{M} \text { a.s. }
\end{aligned}
$$

as $n \rightarrow \infty$ for any integer $r \geq 1$. First, since $\gamma_{M}$ is symmetric, we know $\int x^{2 k-1} d \gamma_{M}=0$ for any integer $k \geq 1$. Second, recalling $\mathbf{U}_{n}$ in (2.10), Proposition 4.13 in [6] says that

$$
\frac{1}{n} E \operatorname{tr}\left(\left(n^{-1 / 2} \mathbf{U}_{n}\right)^{2 k}\right) \rightarrow \int_{\mathbb{R}} x^{2 k} d \gamma_{M}
$$

as $n \rightarrow \infty$ for any $k \geq 1$. Consequently, the two facts together with Lemmas 2.7 and 2.8 yield (2.18).

Proof of Theorem 1. Let $\mu_{n}=p_{n}$ and $\sigma_{n}=\sqrt{p_{n}\left(1-p_{n}\right)}$. Recalling $\boldsymbol{\Delta}_{n}$ in (1.2), $\eta_{i j}^{(n)}$ in $(2.1)$ and $\boldsymbol{\Psi}_{n}$ in (2.2), we know

$$
\boldsymbol{\Delta}_{n}=\underbrace{\sigma_{n} \boldsymbol{\Psi}_{n}+\left(n \mu_{n}\right) \mathbf{I}_{n}}_{\boldsymbol{\Delta}_{n, 1}}-\mu_{n} \mathbf{J}_{n}
$$

where $\mathbf{I}_{n}$ is the $n \times n$ identity matrix, and $\mathbf{J}_{n}$ is the $n \times n$ matrix with all of its entries equal to 1 . By Lemma 2.9, with probability one,

$$
F^{\Psi_{n} / \sqrt{n}} \text { converges weakly to } \gamma_{M}
$$

as $n \rightarrow \infty$. Therefore,

$$
F^{\left(\boldsymbol{\Delta}_{n, 1}-n \mu_{n} \mathbf{I}_{n}\right) / \sqrt{n} \sigma_{n}}=F^{\boldsymbol{\Psi}_{n} / \sqrt{n}} \text { converges weakly to } \gamma_{M}
$$

almost surely as $n \rightarrow \infty$. By (2.19) and the rank inequality (see Lemma 2.2 from [2]),

$$
\begin{aligned}
& \left\|F^{\left(\boldsymbol{\Delta}_{n}-n \mu_{n} \mathbf{I}_{n}\right) / \sqrt{n} \sigma_{n}}-F^{\left(\boldsymbol{\Delta}_{n, 1}-n \mu_{n} \mathbf{I}_{n}\right) / \sqrt{n} \sigma_{n}}\right\| \leq \frac{1}{n} \cdot \operatorname{rank}\left(\frac{\boldsymbol{\Delta}_{n}-\boldsymbol{\Delta}_{n, 1}}{\sqrt{n} \sigma_{n}}\right) \\
\leq & \frac{1}{n} \cdot \operatorname{rank}\left(\mathbf{J}_{n}\right) \leq \frac{1}{n} \rightarrow 0,
\end{aligned}
$$

as $n \rightarrow \infty$, where $\|f\|:=\sup _{x \in \mathbb{R}}|f(x)|$ is the supremum norm of a bounded, measurable function $f(x)$ defined on $\mathbb{R}$. Therefore, (2.20) and (2.21) lead to that, as $n \rightarrow \infty$,

$$
\frac{1}{n} \sum_{i=1}^{n} I\left(\frac{\lambda_{i}\left(\boldsymbol{\Delta}_{n}\right)-n p_{n}}{\sqrt{n p_{n}\left(1-p_{n}\right)}} \leq x\right)=F^{\left(\boldsymbol{\Delta}_{n}-n \mu_{n} \mathbf{I}_{n}\right) / \sqrt{n} \sigma_{n}}(x) \text { converges weakly to } \gamma_{M}
$$

with probability one. The proof is complete. 


\section{Proof of Theorem 2}

For any two probability measures $\mu$ and $\nu$ on $\mathbb{R}$, define

$$
d_{B L}(\mu, \nu)=\sup \left\{\left|\int f d \mu-\int f d \nu\right|:\|f\|_{\infty}+\|f\|_{L} \leq 1\right\},
$$

where $\|f\|_{\infty}=\sup _{x \in \mathbb{R}}|f(x)|,\|f\|_{L}=\sup _{x \neq y}|f(x)-f(y)| /|x-y|$. It is well known (see, e.g., Section 11.3 from [16]) that $d_{B L}(\cdot, \cdot)$ is called the bounded Lipschitz metric, which characterizes the weak convergence of probability measures. Given $n \times n$ real and symmetric matrices $\mathbf{M}_{1}$ and $\mathbf{M}_{2}$, let $\hat{\mu}\left(\mathbf{M}_{i}\right)$ be the empirical measure of the eigenvalues (the spectral measure) of $\mathbf{M}_{i}$ for $i=1,2$, that is,

$$
\hat{\mu}\left(\mathbf{M}_{i}\right)=\frac{1}{n} \sum_{j=1}^{n} \delta_{\lambda_{j}\left(\mathbf{M}_{i}\right)}, \quad i=1,2,
$$

where $\delta_{x}$ is the point mass probability measure at $x$. We have (see, e.g., (2.16) from [6])

$$
d_{B L}^{2}\left(\hat{\mu}\left(\mathbf{M}_{1}\right), \hat{\mu}\left(\mathbf{M}_{2}\right)\right) \leq \frac{1}{n} \operatorname{tr}\left(\left(\mathbf{M}_{1}-\mathbf{M}_{2}\right)^{2}\right) .
$$

A similar result is the Difference Inequality in Lemma 2.3 from [2]:

$$
L^{3}\left(F^{\mathbf{M}_{1}}, F^{\mathbf{M}_{2}}\right) \leq \frac{1}{n} \operatorname{tr}\left(\mathbf{M}_{1}-\mathbf{M}_{2}\right)^{2}
$$

where $F^{\mathbf{M}_{i}}$ is the empirical cumulative distribution function of the eigenvalues of $\mathbf{M}_{i}$ for $i=1,2$. For each $n \geq 2$, recall from (1.4) that $\left\{\xi_{i j}^{(n)} ; 1 \leq i<j \leq n\right\}$ are i.i.d. $\operatorname{Ber}\left(p_{n}\right)$-distributed random variables, and $\xi_{i i}^{(n)}=0$ and $\xi_{i j}^{(n)}=\xi_{j i}^{(n)}$ for all $1 \leq i \neq j \leq n$. Define

$$
\begin{aligned}
& r_{n, i}=\sum_{j=1}^{n} \xi_{i j}^{(n)}, \quad \tilde{r}_{n, i}=r_{n, i}-(n-1) p_{n} \\
& a_{n}=\max _{1 \leq i \leq n}\left\{\left|\tilde{r}_{n, i}\right|\right\} \text { and } b_{n}=\min _{1 \leq i \leq n}\left\{r_{n, i}\right\} .
\end{aligned}
$$

With this notation, we have the following lemma.

LEMMA 3.1 Let $\mathbf{A}_{n}$ and $\mathbf{L}_{n}$ be as in (1.1) and (1.3), respectively. Set $\tilde{\mathbf{L}}_{n}=\mathbf{I}_{n}-\left((n-1) p_{n}\right)^{-1} \mathbf{A}_{n}$. Then

$$
\frac{1}{n} \operatorname{tr}\left(\tilde{\mathbf{L}}_{n}-\mathbf{L}_{n}\right)^{2} \leq \frac{2 a_{n}^{4}+8 n^{2} p_{n}^{2} a_{n}^{2}}{n(n-1)^{4} p_{n}^{4} b_{n}^{2}} \sum_{1 \leq i \neq j \leq n} \xi_{i j}^{(n)}
$$

for all $n \geq 2$.

Proof. If $b_{n}=0$, the conclusion holds trivially. Now, without loss of generality, assume that $b_{n}>0$ for all $n \geq 2$. Note that

$$
\begin{aligned}
\tilde{\mathbf{L}}_{n}-\mathbf{L}_{n} & =-\frac{1}{(n-1) p_{n}} \mathbf{A}_{n}+\operatorname{diag}\left(r_{n, 1}^{-1 / 2}, \cdots, r_{n, n}^{-1 / 2}\right) \mathbf{A}_{n} \operatorname{diag}\left(r_{n, 1}^{-1 / 2}, \cdots, r_{n, n}^{-1 / 2}\right) \\
& =\left(\left(-\frac{1}{(n-1) p_{n}}+\frac{1}{\sqrt{r_{n, i}} \sqrt{r_{n, j}}}\right) \xi_{i j}^{(n)}\right)_{n \times n} .
\end{aligned}
$$


Thus, using $\left(\xi_{i j}^{(n)}\right)^{2}=\xi_{i j}^{(n)}$, we get

$$
\operatorname{tr}\left(\tilde{\mathbf{L}}_{n}-\mathbf{L}_{n}\right)^{2}=\sum_{1 \leq i, j \leq n}\left(\frac{1}{(n-1) p_{n}}-\frac{1}{\sqrt{r_{n, i}} \sqrt{r_{n, j}}}\right)^{2} \xi_{i j}^{(n)} .
$$

Now

$$
\left(\frac{1}{(n-1) p_{n}}-\frac{1}{\sqrt{r_{n, i}} \sqrt{r_{n, j}}}\right)^{2}=\frac{\left(\sqrt{r_{n, i} r_{n, j}}-(n-1) p_{n}\right)^{2}}{(n-1)^{2} p_{n}^{2} r_{n, i} r_{n, j}} .
$$

Use formula $|\sqrt{x}-\sqrt{y}|=|x-y| /(\sqrt{x}+\sqrt{y}) \leq|x-y| / \sqrt{y}$ for $x=r_{n, i} r_{n, j}$ and $y=(n-1)^{2} p_{n}^{2}$ to obtain that the right hand side of (3.6) is dominated by

$$
\frac{\left(r_{n, i} r_{n, j}-(n-1)^{2} p_{n}^{2}\right)^{2}}{(n-1)^{4} p_{n}^{4} r_{n, i} r_{n, j}} \leq \frac{\left(r_{n, i} r_{n, j}-(n-1)^{2} p_{n}^{2}\right)^{2}}{(n-1)^{4} p_{n}^{4} b_{n}^{2}} .
$$

From the definition of $\tilde{r}_{n, i}$, we see that

$$
\begin{aligned}
\left|r_{n, i} r_{n, j}-(n-1)^{2} p_{n}^{2}\right| & =\left|\left(\tilde{r}_{n, i}+(n-1) p_{n}\right)\left(\tilde{r}_{n, j}+(n-1) p_{n}\right)-(n-1)^{2} p_{n}^{2}\right| \\
& =\left|\tilde{r}_{n, i} \cdot \tilde{r}_{n, j}+(n-1) p_{n}\left(\tilde{r}_{n, i}+\tilde{r}_{n, j}\right)\right| \\
& \leq a_{n}^{2}+2(n-1) p_{n} a_{n} .
\end{aligned}
$$

It follows from the inequality $(x+y)^{2} \leq 2 x^{2}+2 y^{2}$ for any $x, y \in \mathbb{R}$ that

$$
\left(r_{n, i} r_{n, j}-(n-1)^{2} p_{n}^{2}\right)^{2} \leq\left(a_{n}^{2}+2(n-1) p_{n} a_{n}\right)^{2} \leq 2 a_{n}^{4}+8 n^{2} p_{n}^{2} a_{n}^{2} .
$$

Thus, collecting all the assertions above, we see that the coefficient of $\xi_{i j}^{(n)}$ in the sum in (3.5) is bounded by

$$
\frac{2 a_{n}^{4}+8 n^{2} p_{n}^{2} a_{n}^{2}}{(n-1)^{4} p_{n}^{4} b_{n}^{2}}
$$

for all $1 \leq i<j \leq n$ and $n \geq 2$. This yields the desired inequality.

We need the following result.

LeMMA 3.2 Let $\epsilon_{1}, \cdots, \epsilon_{n}$ be i.i.d. random variables with $P\left(\epsilon_{1}=1\right)=1-P\left(\epsilon_{1}=0\right)=p \in(0,1)$. Then

$$
P\left(\left|\frac{\sum_{i=1}^{n} \epsilon_{i}}{n p}-1\right| \geq \delta\right) \leq 2 e^{-(n p) \delta^{2} / 4}
$$

for all $n \geq 1$ and $\delta \in(0,1)$.

When $p$ is a constant, that is, $p$ does not depend on $n$, the above is a simple application of the Chernoff bound (see, e.g., p.27 from [14]). In fact, the above result captures the behavior when $p=p_{n}$ goes to 0.

LEMmA 3.3 Assume (1.4) holds. Let $a_{n}$ and $b_{n}$ be as in (3.4). If $n p_{n} / \log n \rightarrow \infty$, then

$$
\frac{a_{n}}{n p_{n}} \rightarrow 0 \text { a.s., } \quad \frac{b_{n}}{n p_{n}} \rightarrow 1 \text { a.s. and } \frac{1}{n^{2} p_{n}} \sum_{1 \leq i \neq j \leq n} \xi_{i j}^{(n)} \rightarrow 1 \text { a.s. }
$$

as $n \rightarrow \infty$. 
Proof. We first prove the last limit. Since $\xi_{i j}^{(n)}=\xi_{j i}^{(n)}$ for all $1 \leq i<j \leq n$, to show the third assertion in the lemma, it is enough to prove that

$$
Z_{n}:=\frac{2}{n(n-1) p_{n}} \sum_{1 \leq i<j \leq n} \xi_{i j}^{(n)} \rightarrow 1 \text { a.s. }
$$

as $n \rightarrow \infty$. Taking $\delta \in(0,1)$, since $n p_{n} / \log n \rightarrow \infty$, we obtain from Lemma 3.2 that $P\left(\left|Z_{n}-1\right| \geq\right.$ $\delta)=O\left(n^{-2}\right)$ as $n \rightarrow \infty$. This shows that $\sum_{n \geq 2} P\left(\left|Z_{n}-1\right| \geq \delta\right)<\infty$, which implies $Z_{n} \rightarrow 1$ a.s. by the Borel-Cantelli lemma.

Recall that $r_{n, i}$ in (3.4) is a sum of $n-1$ i.i.d. $\operatorname{Ber}\left(p_{n}\right)$-distributed random variables for each $1 \leq i \leq n$. Also, $\tilde{r}_{n, i}=r_{n, i}-(n-1) p_{n}, a_{n}=\max _{1 \leq i \leq n}\left\{\left|\tilde{r}_{n, i}\right|\right\}$ and $b_{n}=\min _{1 \leq i \leq n}\left\{r_{n, i}\right\}$. By Lemma 3.2 again, use condition $n p_{n} / \log n \rightarrow \infty$ to get

$$
P\left(\frac{a_{n}}{(n-1) p_{n}} \geq \delta\right) \leq n \cdot P\left(\left|\frac{\sum_{j=2}^{n} \xi_{1 j}}{(n-1) p_{n}}-1\right| \geq \delta\right) \leq \frac{1}{n^{2}}
$$

for any $\delta \in(0,1)$ as $n$ (depending on $\delta)$ is sufficiently large. It follows that $\sum_{n \geq 2} P\left(a_{n} /(n-1) p_{n} \geq \delta\right)$ is finite for any $\delta \in(0,1)$. By the Borel-Cantelli lemma again, we have

$$
\frac{a_{n}}{n p_{n}} \rightarrow 0 \text { a.s. }
$$

as $n \rightarrow \infty$. Finally, from (3.7),

$$
\left|\frac{b_{n}}{(n-1) p_{n}}-1\right|=\left|\frac{\min _{1 \leq i \leq n}\left\{r_{n, i}-(n-1) p_{n}\right\}}{(n-1) p_{n}}\right| \leq \frac{a_{n}}{(n-1) p_{n}} \rightarrow 0 \text { a.s. }
$$

as $n \rightarrow \infty$. This leads to $b_{n} /\left(n p_{n}\right) \rightarrow 1$ a.s. as $n \rightarrow \infty$.

Proof of Theorem 2. Review from Lemma 3.1 that $\tilde{\mathbf{L}}_{n}=\mathbf{I}_{n}-\left((n-1) p_{n}\right)^{-1} \mathbf{A}_{n}$, and $\hat{\mu}\left(\tilde{\mathbf{L}}_{n}\right)$ and $\hat{\mu}\left(\mathbf{L}_{n}\right)$ are the empirical measures of the eigenvalues of $\tilde{\mathbf{L}}_{n}$ and $\mathbf{L}_{n}$, respectively. By Lemma 3.1,

$$
d_{B L}^{2}\left(\hat{\mu}\left(\mathbf{L}_{n}\right), \hat{\mu}\left(\tilde{\mathbf{L}}_{n}\right)\right) \leq \frac{1}{n} \operatorname{tr}\left(\mathbf{L}_{n}-\tilde{\mathbf{L}}_{n}\right)^{2} \leq \frac{2 a_{n}^{4}+8 n^{2} p_{n}^{2} a_{n}^{2}}{n(n-1)^{4} p_{n}^{4} b_{n}^{2}} \sum_{1 \leq i \neq j \leq n} \xi_{i j}^{(n)}
$$

for $n \geq 2$. Set

$$
a_{n}=\left(n p_{n}\right) u_{n}, \quad b_{n}=\left(n p_{n}\right) v_{n} \text { and } \sum_{1 \leq i \neq j \leq n} \xi_{i j}^{(n)}=n^{2} p_{n} w_{n}
$$

for $n \geq 2$. By Lemma 3.3, $u_{n} \rightarrow 0$ a.s., $v_{n} \rightarrow 1$ a.s. and $w_{n} \rightarrow 1$ a.s. as $n \rightarrow \infty$. Therefore, noting $n p_{n} \rightarrow \infty$, we obtain

$$
\begin{aligned}
\frac{2 a_{n}^{4}+8 n^{2} p_{n}^{2} a_{n}^{2}}{n(n-1)^{4} p_{n}^{4} b_{n}^{2}} \sum_{1 \leq i \neq j \leq n} \xi_{i j}^{(n)} & =\frac{2 n^{4} p_{n}^{4} u_{n}^{4}+8 n^{4} p_{n}^{4} u_{n}^{2}}{n^{3}(n-1)^{4} p_{n}^{6} v_{n}^{2}} \cdot n^{2} p_{n} w_{n} \\
& \sim \frac{\left(2 u_{n}^{4}+8 u_{n}^{2}\right) w_{n}}{\left(n p_{n}\right) v_{n}^{2}}=o\left(\frac{1}{n p_{n}}\right) \text { a.s. }
\end{aligned}
$$

as $n \rightarrow \infty$. Therefore,

$$
\frac{1}{n} \operatorname{tr}\left(\mathbf{L}_{n}-\tilde{\mathbf{L}}_{n}\right)^{2}=o\left(\frac{1}{n p_{n}}\right) \text { a.s. }
$$


as $n \rightarrow \infty$. It is easy to see from (3.2) that

$$
d_{B L}^{2}\left(\hat{\mu}\left(\gamma \mathbf{L}_{n}+\rho \mathbf{I}_{n}\right), \hat{\mu}\left(\gamma \tilde{\mathbf{L}}_{n}+\rho \mathbf{I}_{n}\right)\right) \leq \gamma^{2} \cdot \frac{1}{n} \operatorname{tr}\left(\tilde{\mathbf{L}}_{n}-\mathbf{L}_{n}\right)^{2}
$$

for $n \geq 2$ and any two real numbers $\gamma$ and $\rho$. The above two assertions together with (3.8) conclude that

$$
d_{B L}^{2}\left(\hat{\mu}\left(\gamma_{n} \mathbf{L}_{n}+\rho_{n} \mathbf{I}_{n}\right), \hat{\mu}\left(\gamma_{n} \tilde{\mathbf{L}}_{n}+\rho_{n} \mathbf{I}_{n}\right)\right)=o\left(\frac{\gamma_{n}^{2}}{n p_{n}}\right) \quad \text { a.s. }
$$

as $n \rightarrow \infty$ for any two sequences of constants $\left\{\gamma_{n}, n \geq 1\right\}$ and $\left\{\rho_{n}, n \geq 1\right\}$. Notifying $\tilde{\mathbf{L}}_{n}=$ $\mathbf{I}_{n}-\left((n-1) p_{n}\right)^{-1} \mathbf{A}_{n}$, it is clear that

$$
(n-1) p_{n}\left(1-\lambda_{i}\left(\tilde{\mathbf{L}}_{n}\right)\right)=\lambda_{i}\left(\mathbf{A}_{n}\right)
$$

for $i=1,2, \cdots, n$. From the given condition, $\alpha_{n}:=\left(n p_{n}\left(1-p_{n}\right)\right)^{1 / 2} \rightarrow \infty$. By corollary 1.2 from [15], with probability one, $\hat{\mu}\left(\mathbf{A}_{n} / \alpha_{n}\right)$ converges weakly to the semi-circle law $\mu_{c i r}$ with density $\frac{1}{2 \pi} \sqrt{4-x^{2}} I(|x| \leq 2)$. Thus, by (3.10), almost surely,

$$
\frac{1}{n} \sum_{i=1}^{n} I\left(\frac{(n-1) p_{n}}{\alpha_{n}}\left(1-\lambda_{i}\left(\tilde{\mathbf{L}}_{n}\right)\right) \leq x\right), \quad x \in \mathbb{R},
$$

converges weakly to $\mu_{\text {cir }}$ as $n \rightarrow \infty$. Observe that

$$
\frac{(n-1) p_{n}}{\alpha_{n}}=\frac{(n-1) p_{n}}{\left(n p_{n}\left(1-p_{n}\right)\right)^{1 / 2}} \sim \sqrt{\frac{n p_{n}}{1-p_{n}}}
$$

as $n \rightarrow \infty$. By a trivial manipulation, we obtain that, with probability one,

$$
\frac{1}{n} \sum_{i=1}^{n} I\left(\sqrt{\frac{n p_{n}}{1-p_{n}}}\left(1-\lambda_{i}\left(\tilde{\mathbf{L}}_{n}\right)\right) \leq x\right), \quad x \in \mathbb{R},
$$

converges weakly to $\mu_{\text {cir }}$ as $n \rightarrow \infty$. Taking $\gamma_{n}=-\rho_{n}=-\sqrt{n p_{n} /\left(1-p_{n}\right)}$, we have

$$
\lambda_{i}\left(\gamma_{n} \tilde{\mathbf{L}}_{n}+\rho_{n} \mathbf{I}_{n}\right)=\sqrt{\frac{n p_{n}}{1-p_{n}}}\left(1-\lambda_{i}(\tilde{\mathbf{L}})\right)
$$

for $1 \leq i \leq n$. Thus, by (3.11), with probability one, $\hat{\mu}\left(\gamma_{n} \tilde{\mathbf{L}}_{n}+\rho_{n} \mathbf{I}_{n}\right)$ converges weakly to $\mu_{c i r}$ as $n \rightarrow \infty$. Equivalently,

$$
d_{B L}\left(\hat{\mu}\left(\gamma_{n} \tilde{\mathbf{L}}_{n}+\rho_{n} \mathbf{I}_{n}\right), \mu_{c i r}\right) \rightarrow 0 \text { a.s. }
$$

as $n \rightarrow \infty$. By the condition that $\sup \left\{p_{n} ; n \geq 2\right\}<1$, we know $o\left(\gamma_{n}^{2} /\left(n p_{n}\right)\right)=o(1)$ as $n \rightarrow \infty$. Since $d_{B L}(\cdot, \cdot)$ is a metric, by the triangle inequality, we finally conclude from (3.9) and (3.12)that

$$
d_{B L}\left(\hat{\mu}\left(\gamma_{n} \mathbf{L}_{n}+\rho_{n} \mathbf{I}_{n}\right), \mu_{c i r}\right) \rightarrow 0 \text { a.s. }
$$

as $n \rightarrow \infty$. Equivalently, with probability one,

$$
\frac{1}{n} \sum_{i=1}^{n} I\left(\sqrt{\frac{n p_{n}}{1-p_{n}}}\left(1-\lambda_{i}\left(\mathbf{L}_{n}\right)\right) \leq x\right), \quad x \in \mathbb{R},
$$

converges weakly to $\mu_{\text {cir }}$ as $n \rightarrow \infty$.

Acknowledgement. The author thanks Dr. Xue Ding for very helpful discussions on the proof of Theorem 1. 


\section{References}

[1] Z. D. Bai and J. Silverstein, Spectral Analysis of Large Dimensional Random Matrices. Science Press (2006).

[2] Z. D. Bai, Methodologies in spectral analysis of large dimensional random matrices, a review. Statistica Sinica 9 (1999), 611-677.

[3] M. Bauer and O. Golinelli, Random incidence matrices: moments of the spectral density. J. Stat. Phys. 103 (2001), 301-337.

[4] N. L. Biggs, E. K. Lloyd and R. J. Wilson, Graph Theory 1736-1936. Clarendon Press, Oxford (1976).

[5] B. Bollobás, Random Graphs. Academic Press, New York (1985).

[6] W. Bryc, A. Dembo and T. Jiang, Spectral measure of large random Hankel, Markov and Toeplitz matrices. Ann. Probab. 34 (2006), 1-38.

[7] Y. Chow and H. Teicher, Probability Theory, Independence, Interchangeability, Martingales. Springer and New York (1998), 2nd Ed.

[8] F. Chung, Spectral Graph Theory (CBMS Regional Conference Series in Mathematics, No. 92). American Mathematical Society (1997).

[9] F. Chung and L. Lu, Complex Graphs and Networks (CBMS Regional Conference Series in Mathematics, No. 107). American Mathematical Society (2006).

[10] F. Chung, L. Lu and V. Vu, Eigenvalues of random power law graphs. Annals of Combinatorics 7 (2003), 21-33.

[11] F. Chung, L. Lu and V. Vu, The spectra of random graphs with given expected degrees. Proceedings of National Academy of Science, 100 (2003), 6313-6318.

[12] Y. Colin de Verdière, Spectres de Graphes. Societe Mathematique De France (1998).

[13] D. Cvetković, M. Doob and H. Sachs, Spectra of Graphs: Theory and Applications. Johann Ambrosius Barth, Heidelberg (1995), 3rd ed.

[14] A. Dembo and O. Zeitouni, Large Deviations Techniques and Applications. Springer (1998), 2nd edition.

[15] X. Ding and T. Jiang, Spectral distributions of adjacency and Laplacian matrices of random graphs. Ann. Appl. Probab. 20 (2010), 2086-2117.

[16] R. Dudley, Real Analysis and Probability. Cambridge University Press (2002).

[17] P. Erdös and A. Rényi, On the strength of connectedness of a random graph. Acta Math. Acad. Sci. Hungar. 12 (1961b), 261-267.

[18] P. Erdös and A. Rényi, On the evolution of random graphs. Bull. Inst. Int. Statist. Tokyo 38 (1961a), 343-347.

[19] P. Erdös and A. Rényi, On the evolution of random graphs. Magyar Tud. Akad. Mat. Kuató Int. Közl 5 (1960), 17-61.

[20] P. Erdös and A. Rényi, On random graphs I. Publ. Math. Debrecen 6 (1959), 290-297. 
[21] S. N. Evangelou, A numerical studies of sparse random matrices. J. Stat. Phys. 69 (1992), 361-383.

[22] S. N. Evangelou, Quantum percolation and Anderson transition in dilute system. Phys. Rev. B. 27 (1983), 1397-1400.

[23] S. N. Evangelou and E. N. Economou, Spectral density singularities, level statistics, and localization in sparse random matrices. Phys. Rev. Lett. 68 (1992), 361-364.

[24] P. Forrester, Log-Gases and Random Matrices. Princeton University Press (2010).

[25] Y. V. Fyodorov and A. D. Mirlin, Universality of the level correlation function of sparse random matrices. J. Phys. A. 24 (1991), 2273-2286.

[26] A. Juozulynas, The eigenvalues of very sparse random symmetric matrices. Lithuanian Math. J. 44 (2004), 62-70.

[27] L. Khorunzhiy, W. Kirsch and P. Müller, Lifshitz tails for spectra of Erdös-Rényi random graphs. Ann. Appl. Probab. 16 (2006), 295-309.

[28] A. Khorunzhy and G. J. Rodgers, Eigenvalue distribution of large dilute random matrices. J. Math. Phys. 38 (1997), 3300-3320.

[29] O. Khorunzhy, M. Shcherbina and V. Vengerovsky, Eigenvalue distribution of large weighted random graphs. J. Math. Phys. 45 (2004), 1648-1672.

[30] G. Kirchhoff, Über die Aufösung der Gleichungen, auf welche man bei der untersuchung der linearen verteilung galvanischer Ströme geföhrt wird. Ann. Phys. Chem. 72 (1847), 497-508.

[31] M. Krivelevich and B. Sudakov, The largest eigenvalue of sparse random graphs. Combin. Probab. Comput. 12 (2003), 61-72.

[32] R. Kühn, Spectra of sparse random matrices. J. Phys. A: Math. Theor. 41 (2008), 295002.

[33] B. D. Mckay, The expected eigenvalue distribution of a large regular graph. Linear Algebra and Its Appl. 40 (1981), 203-216.

[34] M. Penrose, Random Geometric Graphs (Oxford Studies in Probability). Oxford University Press (2003).

[35] T. Puppe, Spectral Graph Drawing: A Survey. VDM Verlag (2008).

[36] G. J. Rogers and A. J. Bray, Density of states of a sparse random matrix. Phys. Rev. B, 37 (1988), 3557-3562.

[37] G. J. Rogers and C. De Dominicis, Density of states of sparse random matrices. J. Phys. A: Math. Gen. 23 (1990), 1567-1573.

[38] G. Semerjian and L. F. Cugliandolo, Sparse random matrices: the eigenvalue spectrum revisited. J. Phys. A: Math. Gen. 35 (2002), 4837-4851. 